\title{
SEGURIDAD ENERGÉTICA DEL MAGREB ENTRE LA NUEVA ESTRATEGIA \\ ENERGÉTICA EUROPEA Y EL MEDITERRÁNEO ORIENTAL - ORIENTE PRÓXIMO
}

\author{
Eric Pardo ${ }^{1}$ \\ UNISCI / Universidad Complutense de Madrid
}

\begin{abstract}
Resumen:
Con el conflicto en Ucrania, la Unión Europea inició una revisión de su política energética; siendo este conflicto el detonante, entre los objetivos manifiestos, se puede encontrar la reducción de la dependencia del gas natural de Gazprom. Esto podría ser una noticia positiva para los países productores del Magreb, tales como Argelia y Libia. Sin embargo, la diversificación de la Unión Europea hacia países del Mediterráneo Oriental y el Oriente Próximo podría liberar recursos gasísticos adicionales que pudiesen terminar compitiendo con el Magreb. El que la UE compita con las demandas internas de esta región y la competición de otros importadores, hace difícil que ello ocurra; además, en materia de precios, solo Qatar parece representar un serio competidor potencial, si bien, por el momento, no hay perspectivas de que se levante la moratoria sobre producción. Por otra parte, la UE no busca diversificar sus importaciones de petróleo, si bien, las dinámicas del mercado internacional, definidas por el comportamiento de los principales productores de Oriente Próximo, sí está teniendo un serio impacto al mantener los precios bajos, dañando así las finanzas de Argelia y Libia.
\end{abstract}

Palabras clave: estrategia energética de la UE, Argelia, Libia, Magreb, gas natural, petróleo, diversificación.

Title in English: "Energy Security in the Maghreb Facing the New European Energy Strategy and the Eastern Mediterranean - Middle East"

\begin{abstract}
:
The conflict in Ukraine was the trigger for a revision of the European Union's energy policy; the main goal was to reduce dependence on Gazprom's natural gas. Gas producers in the Maghreb region, such as Algeria and Libya, might receive this as good news. However, could diversification towards new partners in the Eastern Mediterranean and the Middle East free new resourses that might compite with natural gas produced in the Maghreb? The EU is at disatvantage in the competition, as it faces both the internal demand in the West Mediterranean and Middle East regions and other world consumers' demand. It is thus unlikely that natural gas from these regions displaces Maghreb gas producers from the EU market in the coming future. Only Qatar represents a serious potential competitor. However, this country does not seem ready to lift its moratorium on natural gas production. On the other hand, while the EU does not seek to diversify its imports of oil, the oil market's evolution, determined by decisions taken by the main producers from the Middle East, does have a serious impact on the Maghreb: OPEC's policy maintains prices low, damaging thus the public finances of Algeria and Libya.
\end{abstract}

Keywords: EU Energy Security Strategy, Algeria, Libya, Maghreb, natural gas, oil, diversification.

Copyright (C) UNISCI, 2015.

Las opiniones expresadas en estos artículos son propias de sus autores, y no reflejan necesariamente la opinión de UNISCI. The views expressed in these articles are those of the authors, and do not necessarily reflect the views of UNISCI.

\footnotetext{
${ }^{1}$ Eric Pardo Sauvageot es doctor por la Universidad Complutense, Departamento de Estudios Internacionales de la Facultad de Ciencias Políticas y Sociología de la Universidad Complutense, es investigador senior de UNISCI y miembro del Foro Hispano-Argelino.

E-mail: epardosauvageot@hotmail.com.
}

http://dx.doi.org/10.5209/rev_RUNI.2015.n39.51810 


\section{Introducción: política energética de la UE}

La guerra en Ucrania y la implicación rusa, tanto en la anexión de la Península de Crimea y Sebastopol, como en el apoyo militar a las fuerzas rebeldes en Donetsk y Lugansk ha influido enormemente en la revisión de la política energética por parte de la UE. Según podemos observar en la página de la Unión Energética (explicada seguidamente), el factor geopolítico figura entre los tres factores (los otros dos son el inicio de la nueva Comisión y la revisión de los objetivos que dan sentido a tal Unión) $)^{2}$. Qué duda cabe que son los sucesos en Ucrania los que se encuentran detrás de ello ${ }^{3}$. Sin ir más lejos, lo recordaba recientemente el Comisario de Acción por el Clima y Energía, Miguel Arias Cañete, en un artículo de opinión en el diario El País:

"Durante mucho tiempo hemos dado por sentado que este suministro era fiable, pero acontecimientos recientes en el este de Europa han demostrado lo contrario" ... "Europa seguirá necesitando importar parte de su energía, sobre todo gas, pero tenemos que diversificar la lista de proveedores" ${ }^{4}$

No es casual que las primeras llamadas a crear una Unión Energética viniesen por parte de Polonia $^{5}$, cuyo posicionamiento en política exterior es particularmente beligerante en relación con la Federación Rusa. Los planteamientos del entonces primer ministro polaco (y actual Presidente de la Unión Europea), Donald Tusk, según los cuales tal unión había de plantearse ante todo con el fin de mejorar las interconexiones europeas y estar preparados para afrontar interrupciones en el suministro de gas natural eran muy oportunos. No hay que olvidar los efectos de la disputa energética de enero del 2009 sobre los países más vulnerables de la Unión Europea (véase en particular, Bulgaria), ${ }^{6}$ máxime teniendo en cuenta que tal vulnerabilidad persiste ${ }^{7}$.

De hecho, las sospechas de que el empeoramiento de las relaciones Rusia-Ucrania pusiese en peligro los suministros, estaban plenamente justificada, pues del 16 de junio al 2 de diciembre, los suministros de Gazprom a Ucrania quedaron suspendidos ante la imposibilidad de resolver una disputa sobre la deuda del monopolio energético ucraniano, Naftohaz, y sobre el precio de los suministros ${ }^{8}$. Menos oportuna quizá era la idea de hacer funcionar a la hipotética unión energética como un frente único a la hora de negociar precios con Gazprom, como ya avanzaba días antes Van Rompuy ${ }^{9}$, como concretaría el mismo Tusk días más tarde ${ }^{10}$

\footnotetext{
2 "Energy Union", en http://ec.europa.eu/energy/en/topics/energy-strategy/energy-union.

3 En la Estrategia Europea de Seguridad Energética de mayo del 2014, en http://eur-lex.europa.eu/legalcontent/EN/ALL/?uri=CELEX:52014DC0330\&qid=1407855611566, la mención a las disputas energéticas entre Rusia y Ucrania de enero del 2006 y 2009 prácticamente abre el documento (p. 2), al igual que se distingue a Rusia a la hora de hablar de la dependencia energética en gas natural (p. 2).

${ }^{4}$ Arias Cañete, Miguel Ángel: "La energía nos concierne", El País, 26 de febrero 2015, en http://elpais.com/elpais/2015/02/25/opinion/1424878009 695939.html.

5 "Poland calls for EU energy union", Euractiv, 2 de abril 2014, en http://www.euractiv.com/sections/energy/poland-calls-eu-energy-union-301303.

${ }_{6}^{6}$ Kovacevic Aleksandar: "The Impact of the Russia-Ukraine Gas Crisis in South-Eastern Europe", Oxford Institute for Energy Studies, NG 29 (Marzo 2009), en http://www.oxfordenergy.org/2009/03/the-impact-of-therussia $\% \mathrm{E} 2 \% 80 \% 93$ ukraine-gas-crisis-in-south-eastern-europe/.

${ }^{7}$ Sharples, Jack and Judge, Andy: "Bulgaria and Macedonia would be hardest hit by a suspension of Russian gas exports through Ukraine", LSE Europp Blog (24 de marzo 2014), en

http://blogs.lse.ac.uk/europpblog/2014/03/13/bulgaria-macedonia-and-romania-would-be-hardest-hit-by-asuspension-of-russian-gas-exports-through-ukraine/ y versión más larga en: http://gpf-europe.com/forum/?page=post\&blog=energy\&id=157.

${ }^{8}$ Ver los artículos publicados por Eric Pardo en el blog Eurasianet sobre tal disputa en: http://eurasianet.es/author/epardo/.

9 "EU plans to reduce Russian energy dependence", Euractiv, 21 de marzo 2014, en
} 
y en la dirección por la que la Comisión pocos meses después decidía ir $^{11}$; si bien absolutamente justificado desde un estricto punto de vista económico, parece indicar un particular abuso por parte de Gazprom en su política de precios, una lectura de la realidad, al menos parcialmente, inexacta. De hecho, el objetivo declarado de ofrecer:

"Una transición energética que garantice energía asequible, segura, competitiva y sostenible para todos" ${ }^{\prime \prime 2}$

Podría verse puesto en cuestión, en lo que al primer adjetivo (en negrita) se refiere, por el empeño en reducir la dependencia de la Federación Rusa y de Gazprom.

En todo caso, hay que entender que ya en la cumbre del 21 al 22 de marzo de 2014, los líderes de la Unión Europea habían puesto el acento sobre la dependencia energética y los desafíos que de ello se derivaban ${ }^{13}$. De hecho, la publicación en mayo de 2014 de una estrategia de seguridad energética ${ }^{14}$ era el resultado de tal cumbre. En ella cabe distinguir dos objetivos en particular: mejorar la resistencia de la UE a cortes de flujo y diversificar los suministros exteriores y las infraestructuras relacionadas ${ }^{15}$. Como comunicó el VicePresidente de la Comisión Europea al cargo de la Unión Energética el 25 de febrero de 2015 al Parlamento Europeo, la Comisión había publicado su proyecto de Unión Energética ${ }^{16}$.

Como podemos ver en la estrategia europea, las dimensiones identificadas son: seguridad energética, solidaridad y confianza, un mercado energético europeo plenamente integrado, eficiencia energética que contribuya a la moderación de la demanda, la descarbonización de la demanda e Investigación, innovación y competitividad ${ }^{17}$. De particular interés para nosotros es el apartado de seguridad energética y su implicación sobre la diversificación energética. En este sentido, la comunicación presentando el paquete de una unión energética es explícita cuando anuncia:

"The political challenges over the last months (véase, Ucrania) have shown that diversification of energy sources, suppliers and routes is crucial for ensuring secure and resilient energy supplies to European citizens and companies" ${ }^{\prime 18}$

Indudablemente, aparte de las medidas que se proponen para integrar más el mercado interior de la UE, que necesariamente tienen importancia siempre que haya perturbaciones en el flujo de gas natural, una mejora de la seguridad energética se daría con una mayor diversificación y con una mayor prevención de los elementos presentes en las turbulentas relaciones energética Rusia-Ucrania; lo primero minimizaría el impacto de cualquier disputa, mientras que lo segundo simplemente reduciría el riesgo mismo de que disputas que afecten el tránsito,

http://www.euractiv.com/energy/eu-leaders-discuss-reducing-ener-news-534344.

${ }^{10}$ Tusk, Donald: "A united Europe can end Russia’s energy stranglehold", Financial Times, 21 de abril 2014, en http://www.ft.com/intl/cms/s/0/91508464-c661-11e3-ba0e-00144feabdc0.html.

11 "Will EU states play ball on Energy Union?", Euractiv, 25 de febrero 2015, en

http://www.euractiv.com/sections/energy/will-eu-states-play-ball-energy-union-312419.

${ }^{12} \mathrm{http}: / /$ europa.eu/rapid/press-release_SPEECH-15-4503_en.htm.

13 "EU plans to reduce Russian energy dependence", Euractiv, 21 de marzo 2014, en

http://www.euractiv.com/energy/eu-leaders-discuss-reducing-ener-news-534344.

${ }^{14}$ Estrategia Europea de Seguridad Energética, op. cit.

${ }_{16}^{15}$ Ibid., p. 3.

16 "Vice-President Šefčovič speech at the European Parliament plenary: Decision adopted on a Strategic framework for the Energy Union", Comisión Europea, Bruselas (25 de febrero 2015), en

http://europa.eu/rapid/press-release SPEECH-15-4503 en.htm.

${ }^{17}$ Estrategia Europea de Seguridad Energética, op. cit., p. 4.

${ }^{18}$ Ibid., p. 4. 
surgiesen. La atención de la Unión Europea parece estar volcada principalmente en el primer elemento. Se mencionan pues las siguientes áreas geográficas como prioritarias como alternativas al gas natural ruso, ya sea como productores o como países de tránsito: Argelia y Turquía, Azerbaiyán y Turkmenistán; Oriente Medio; África y otros suministradores potenciales (ej. EEUU) al tiempo que se recomienda reforzar el vínculo con uno de los principales suministradores a Europa, Noruega ${ }^{19}$.

La cuestión que más cabe resaltar, es que planteada la dependencia energética de la UE de Rusia como un problema, necesariamente se revalorizan una serie de alternativas, como las mencionadas más arriba. En este artículo vamos a analizar una de las alternativas, por lo menos a primera vista, más prometedoras, véase los recursos en gas natural del Mediterráneo Oriental y de Oriente Próximo. Interesa en este artículo valorar cuál es el potencial real de esta región, para determinar qué impacto podría ello tener en el futuro sobre la dependencia de la UE, no ya de Rusia, sino del Magreb mismo. ${ }^{20}$ Como hemos visto en el párrafo superior, lejos de propugnarse una reducción de la dependencia de esta región, con Argelia como principal productor, se apuesta por mantenerla y aumentarla en la medida de lo posible. Sin embargo, la entrada en escena de nuevos recursos provenientes del Mediterráneo Oriental y Oriente Próximo podría sentar las bases para una reducción futura de tal dependencia, si con ello entran en el mercado recursos más competitivos o si ello sirve de polo de atracción de inversiones que podrían en otras circunstancias dirigirse al Magreb.

Por otra parte, no se puede perder de vista un factor que resultará, si cabe, tal y como discutiremos en nuestras conclusiones, más determinante aún para los países del Magreb, y que es la evolución del mercado internacional del petróleo. Analizaremos, así, en qué medida las decisiones adoptadas por países de Oriente Próximo en los últimos meses pueden impactar en nuestros países del Magreb.

\section{Recursos gasísticos en el Mediterráneo Oriental y Oriente Próximo}

\subsection{Chipre, Líbano, Israel}

Entre las alternativas potenciales en el apartado de diversificación energética para la UE, Chipre figura como una de las más prometedoras por su potencial de suministro de gas natural. El principal yacimiento, Afrodita, contendría unas reservas de entre 84254Mmill.mc ${ }^{21}$. El descubrimiento en diciembre de 2011 por la empresa Noble Energy de un yacimiento de gas natural en alta mar en aguas territoriales de Chipre ${ }^{22}$ abría la puerta a un nuevo actor en el complejo juego energético entorno a Europa. Sin embargo, Chipre es un potencial actor que no carece de dificultades para Europa.

En primer lugar, las tensas relaciones con Turquía pueden representar un notable freno al buen desarrollo de la explotación de los yacimientos de gas natural descubiertos. Turquía ha emitido hasta el momento cuatro reservas en cuanto al derecho de la República de Chipre a explotar los recursos: 1/ los recursos habrían de ser compartidos con los turco-chipriotas; 2/ la República chipriota no tiene derecho a negociar contratos de explotación sobre un territorio

\footnotetext{
${ }^{19}$ Ibid., pp. 6-7.

${ }^{20}$ Para la energía en los países del Magreb, ver el artículo en esta edición de Soledad Segoviano.

${ }^{21}$ Derbouche, Hakim; El-Katiri, Laura y Fattouh, Bassam: "East Mediterranean Gas - what kind of a gamechange", Oxford Institute for Energy Studies, NG 71 (Diciembre de 2012), en http://www.oxfordenergy.org/2012/12/east-mediterranean-gas-what-kind-of-a-game-changer/, p. 4.

${ }^{22}$ Glamouridis, Anastasios: "The Offshore Discovery in the Republic of Cyprus - Monetisation Prospects and Challenges", Oxford Institute for Energy Studies, NG 65 (Julio 2012), p. iii, en

http://www.oxfordenergy.org/2012/07/the-offshore-discovery-in-the-republic-of-cyprus-monetisation-prospectsand-challenges/.
} 
que a fin de cuentas no es suyo, sino conjunto con los turco-chipriotas; 3/ el área económica exclusiva de la República de Chipre se solapa sobre la de Turquía y 4/ Se violan los derechos de los turco-chipriotas ${ }^{23}$.

En segundo lugar, se encuentran las dificultades inherentes a la explotación misma de los recursos. El aspecto positivo es que, por un lado, el consumo potencial de Chipre está muy lejos de representar un verdadero competidor para cualquier futuro consumidor: el consumo de gas natural de Chipre para 2025 no debería superar 1,7Mmill.mca ${ }^{24}$. De hecho, la rentabilidad de las explotaciones en alta mar depende necesariamente de su exportación a los mercados más lucrativos. Sin embargo, las dudas llegan a la hora de determinar cuál sería el mejor modo de realizar la exportación, bien en gasoductos o como gas licuado, y de poder asegurarse que una parte sustancial de las exportaciones pudiesen acabar en los mercados europeos, pues Turquía representa un serio competidor. La construcción de un gasoducto a Grecia representa un serio desafío técnico por las profundidades a salvar y dependería de que las interconexiones necesarias fuesen construidas para poder servir más allá de Grecia y por tanto ser plenamente rentable ${ }^{25}$. La opción de Turquía, aunque más rentable, vista la cercanía geográfica, se encontraría con los mismos problemas que la opción griega, pues el mercado turco resultaría insuficiente; sería necesario complementar con el mercado del Sureste Europeo $^{26}$. Más problemático aún es que aun si estas condiciones se pudiesen dar, no prefiriese Chipre optar por establecer terminales de LNG a fin de evitar depender de un solo mercado ${ }^{27}$. Licuar el gas natural ofrece no solo en términos generales la posibilidad de no caer cautivo de un solo mercado, sino de abrirse en particular al pujante mercado asiático, donde la demanda previsiblemente aumentará ${ }^{28}$. Las perspectivas en Europa son más bien de estancamiento $^{29}$, si bien el agotamiento de recursos domésticos deja la puerta abierta para la entrada de nuevos actores.

El elemento de particular interés para la UE, es que no se trata solo de Chipre, sino que la perspectiva es de que en toda la región, incluyendo además países como Líbano, Israel y los Territorios Palestinos, haya un total de 980Mmill.mc de reservas de gas natural, lo cual convierte a esta región en un potencial productor privilegiado dada su cercanía a Europa ${ }^{30}$. Sin embargo, no hay que perder de vista que estos recursos adicionales tienen que contraponerse a las perspectivas de crecimiento de la demanda de los mismos productores y de sus inmediatos vecinos, al igual que la posibilidad de que el excedente de exportación encuentre mejores mercados fuera de Europa.

El primer aspecto muestra que de Israel y Chipre deberían quedar sustanciales volúmenes libres para la exportación. En el caso de Israel, la producción para 2020 debería alcanzar el mayor volumen de los dos países, con una horquilla de 15-20Mmill.mca. Sin embargo, el consumo doméstico debería igualmente aumentar, llegando hasta los 12,5Mmill.mca; ello dejaría libres de 2,5 a 7,5Mmill.mca para la exportación. El caso de

\footnotetext{
${ }^{23}$ Ibid., p. 29.

${ }^{24}$ Ibid., p. 37.

${ }^{25}$ Ibid., p. 43.

${ }^{26}$ Ibid., p. 47.

${ }^{27}$ Ibid., p. 43.

${ }^{28}$ Según un informe del Instituto de Energía de Oxford del 2014, la demanda en Asia debería sobrepasar los 400Mmill.mc. anuales en 2025, mientras que en 2010 se encontraba en unos 180Mmill.mc (Ver: Rogers, Howard y Stern, Jonathan: "Challenges to JCC Pricing in Asian LNG Markets", Oxford Institute for Energy Studies, NG 81 (Febrero 2014), p. 9, gráfico 5, en http://www.oxfordenergy.org/2014/02/challenges-to-jccpricing-in-asian-lng-markets-2/).

${ }^{29}$ Honoré, Anouk: "The Outlook for Natural Gas Demand in Europe", Oxford Institute for Energy Studies, NG 87 (Junio 2014), en http://www.oxfordenergy.org/2014/06/the-outlook-for-natural-gas-demand-in-europe-2/.

${ }^{30}$ Derbouche, El-Katiri y Fattouh, op. cit., p. 2.
} 
Chipre es aún más favorable para el mercado internacional del gas natural, pues las perspectivas de consumo en la república insular son muy modestas: para 2020, la República de Chipre consumiría solo 0,9 de $9 \mathrm{Mmill}$ mca, con lo que prácticamente todo el excedente podría exportarse ${ }^{31}$. Aparte de los excedentes que se prevén, la exportación representa igualmente una necesidad financiera ineludible para la explotación de los nuevos recursos. No hay que perder de vista, sin embargo, que Siria, según se calculaba en 2012, tendría un déficit adicional de $10 \mathrm{Mmill}_{\text {.mca }}{ }^{32}$, con lo cual podría representar un mercado muy sustancial para estos nuevos suministros. La terrorífica situación de guerra civil que sufre todavía en estos momentos el país no nos permite, empero, realizar estimaciones fiables sobre su futuro consumo, que resultará necesariamente mucho menor que lo previsto si la guerra persiste o si no se logra superar rápidamente la devastación provocada. El obstáculo para la UE es que en el contexto actual de bajos precios del gas natural en el mercado internacional, podría resultar inviable cualquier proyecto de LNG desde estos países, a la par que construir gasoductos hacia Europa supondría igualmente un poderoso desafío en cuestión de infraestructuras, y en último término, igualmente de precio ${ }^{33}$. El mercado natural para estos nuevos yacimientos habría de ser Egipto, lo cual, a la vista de los nuevos desarrollos que vemos más abajo, queda igualmente en entredicho.

\subsection{Egipto}

A día de hoy, tras el descubrimiento del yacimiento gigante de "Zohr", es probablemente Egipto el que representa la mayor esperanza. El yacimiento, cuyo descubrimiento ha sido realizado por la italiana ENI, es hasta el momento el de mayores dimensiones, superando, no solo a "Afrodita", con 127Mmill.mc, sino también a los gigantes en aguas territoriales israelíes, "Leviatán" y "Tamar", con 622Mmill.mc y 283Mmill.mc respectivamente, pues "Zohr" podría contar con unas reservas de hasta $843 \mathrm{Mmill.mc}{ }^{34}$ El potencial de este yacimiento es considerable en cuanto a cómo podría impactar el panorama energético regional. Como hemos podido observar más arriba, a pesar del previsible aumento del consumo doméstico en Israel y vista la imposibilidad de Siria para siquiera restablecer su antigua capacidad importadora, sustanciales volúmenes de recursos en LNG Israel y Chipre podrían destinarse al mercado internacional.

Esta posibilidad, en lo que respecta a la política de diversificación de la UE, se vería ensombrecida por las perspectivas de demanda de Egipto, así como la caída de su producción. El endeudamiento del país, especialmente en el inestable contexto de cambio político con la caída de Hosni Mubarak y el proceso revolucionario, representó un freno para aumentar la inversión, con lo que la producción cayó del máximo de 63Mmill.mca en 2009 hasta $56 \mathrm{Mmill}$.mca en $2013^{35}$; esto impactó enormemente en las exportaciones de LNG desde este país, que empezó a exportar gas natural en $2003^{36}$; estas pasaron de cerca de $20 \mathrm{Mmill}$.mca en $2009^{37}$ a solo 3,7Mmill.mca en 2013, de los cuales un simbólico 0,4Mmill.mca habrían alcanzado Europa ${ }^{38}$. De hecho, Egipto parecía estar destinado a convertirse en importador

\footnotetext{
${ }^{31}$ Ibid., p. 19, tabla 7.

${ }^{32}$ Ibid., Idem.

${ }^{33}$ Leigh, Michael: " Eastern Mediterranean Gas Won’t Solve Europe’s Energy Dilemmas", German Marshall Fund (GMF), 16 de diciembre 2014, en http://www.gmfus.org/blog/2014/12/16/eastern-mediterranean-gaswon $\%$ E2\%80\%99t-solve-europe $\%$ E2\%80\%99s-energy-dilemmas y Gyamouridis, op. cit., p. 16.

34 "ENI makes giant discovery in Egyptian Waters", Natural Gas Europe, 31 de agosto 2015, en http://www.naturalgaseurope.com/eni-makes-giant-discovery-in-egyptian-waters-25202.

${ }^{35}$ Ralph Dickel et al., op. cit., p. 18.

${ }^{36}$ Tsafos, Nikos: "Egypt: A Market for Natural Gas from Cyprus and Israel?", German Marshall Fund (GMF), 14 de diciembre 2015, p. 2, en http://www.gmfus.org/publications/egypt-market-natural-gas-cyprus-and-israel.

${ }^{37}$ Ibid., fig. 6, p. 18.

${ }^{38}$ Ibid., table 6, p. 17.
} 
neto de gas natural ${ }^{39}$, apareciendo así como el destino de los nuevos recursos desarrollados en el Mediterráneo Oriental. En este sentido, Egipto firmó en marzo del 2015 un controvertido acuerdo con Israel para importar del orden de 5Mmill.mc durante los tres años de duración del contrato $^{40}$. Ello iba de la mano con la que había sido la tendencia desde el año 2000, cuando Egipto se convirtiese en exportador de LNG, de ver su demanda crecer a mayor ritmo que su producción ${ }^{41}$; con la perspectiva de que la población aumente y con una intensidad energética aún por debajo de la media de la OCDE la demanda necesariamente crecerá ${ }^{42}$.

Con la aparición de "Zohr", existe por tanto la posibilidad, no solo de que Egipto revierta el escenario de convertirse en un país importador, sino que recupere su capacidad exportadora. Aunque lo segundo no llegase a materializarse, tendrían un impacto potencial en la UE, pues liberaría los recursos de Israel y Chipre que podrían haberse visto absorbidos por la demanda egipcia ${ }^{43}$. Sin embargo, cabe al mismo tiempo la posibilidad de que precisamente la competencia de Zohr dé al traste con las perspectivas de desarrollo de Tamar, Leviatán y quizá también Afrodita: Delek y Noble, las empresas responsables de los proyectos en Chipre e Israel se encuentran con que el mercado inicialmente pensado de Egipto puede verse perfectamente sustituido por Zohr, cuyos costes de explotación deberían ser sustancialmente menores y que podría entrar en funcionamiento a partir del mismo 2016; se necesitarían varios años para el desarrollo de los otros yacimientos del Mediterráneo Oriental ${ }^{44}$. Aun cuando la producción de Zohr no llegase a dar abasto para ocupar parte de la demanda europea, los bajos precios del mercado internacional del gas natural en estos momentos suponen un poderoso freno para el desarrollo de unos proyectos a fin de cuentas muy $\operatorname{costosos}^{45}$. Dicho sea de paso, la posibilidad misma de que Zohr sea fuente de exportaciones, se encuentra igualmente constreñida por el estado actual del mercado internacional del gas natural y por las limitaciones que ENI seguramente enfrente para destinar gas natural al

\footnotetext{
${ }^{39}$ Las primeras importaciones de LNG en Egipto empezaron en abril del 2015 (Ver: Fick, Maggie y Dagenborg, Joachim: "UPDATE 2-Egypt reaches deal with Norway's Hoegh on LNG import terminal", Reuters, 12 de mayo 2014, en http://uk.reuters.com/article/2014/05/12/egypt-energy-gas-idUKL6N0NY1YR20140512). Igualmente, se firmó en julio del 2015 un acuerdo con Rosneft para la importación de productos petrolíferos, así como LNG (Véase Tejas, Aditya: "Egypt Signs LNG, Petroleum Import Deal With Russia's Rosneft", International Business Times, 8 de julio 2015, en http://www.ibtimes.com/egypt-signs-lng-petroleum-import-deal-russias-rosneft1998857). Actualmente, Egipto está en conversaciones con Jordania para utilizar el puerto de Aqaba con el fin de importar LNG para su consumo en Egipto (Ver: "Egypt Seeks LNG via Jordan", Oil and Gas Eurasia, 24 de agosto 2015, en https://www.oilandgaseurasia.com/en/news/egypt-seeks-lng-jordan).

40 "Egypt to import gas from Israel via EMG pipeline", Mada Masr, 18 de marzo 2015, en http://www.madamasr.com/news/egypt-import-gas-israel-emg-pipeline.

${ }^{41}$ Ghafar, Adel Abdel: "Egypt's new gas discovery: Opportunities and challenges", Brookings, 10 de septiembre 2015, en http://www.brookings.edu/research/opinions/2015/09/10-egypt-gas-discovery-abdelghafar.

${ }^{42}$ Tsafos, op. cit., p. 3.

${ }^{43}$ Por el momento, Egipto afirma que los planes para importar gas natural de Israel se mantienen, pues los recursos se destinarán principalmente a mejorar la demanda doméstica, poniendo fin a los problemas de suministro en el mercado doméstico ("Egypt will not stop importing gas from Israel after discovery of Zohr gas field", Middle East Monitor, 3 de septiembre 2015, en https://www.middleeastmonitor.com/news/africa/20839egypt-will-not-stop-importing-gas-from-israel-after-discovery-of-zohr-gas-field).

${ }_{44}$ Luft, Gal: "Will ENI's discovery in Egypt sink Israel's Leviathan?", Natural Gas Europe, 1 de septiembre 2015, en http://www.naturalgaseurope.com/eni-zohr-gas-field-egypt-discovery-impacts-israel-leviathan-25193.

${ }^{45}$ La opción de exportar LNG a Europa resultaría demasiado costosa y dañaría la competitividad de tales exportaciones ("Energy security will not cure all of Egypts ills", Natural Gas Europe, en

http://www.naturalgaseurope.com/energy-security-will-not-cure-all-of-egypts-ills-25301), mientras que la única alternativa viable, véase, Europa Oriental (Grecia) y Turquía, carece de las infraestructuras necesarias en materia de LNG, siendo los costes de construcción de nuevos gasoductos demasiado elevados (Ibid.); no hay que perder de vista que según ciertas estimaciones, Egipto habrá logrado volver a la autosuficiencia a partir de la próxima década, con lo que la ventana de oportunidad para Delek y Noble es corta, lo cual ahonda las dudas sobre su viabilidad (ver: Stefanini, Sara: " Egypt's example — EU blesses Shell-BG union", Politico, 9 de marzo 2015, en http://www.politico.eu/article/politico-pros-morning-energy-egypts-example-eu-blesses-shell-bg-union/).
} 
extranjero, frente a los deseos de Egipto ${ }^{46}$. Además, no hay de hacer perder de vista el potencial de disputa con Chipre que este nuevo proyecto contiene, pues los yacimientos de Zohr podrían extenderse hacia la ZEE de Chipre ${ }^{47}$.

\subsection{El Corredor Sur: Nabucco y TAP}

La decisión en junio del 2013, por parte de Azerbaiyán, de destinar sus recursos de gas natural a través del gasoducto TAP (Trans-Adriatic Pipeline) ${ }^{48}$, que conectaría Turquía con Grecia y (pasando por Albania), Italia, y que estaría operativo (se prevé que para 2019) ${ }^{49}$ tan pronto Turquía construya el gasoducto TANAP (Trans-Anatolian Pipeline), dejó sin razón de ser al ambicioso proyecto Nabucco. El sentido de Nabucco era abrir la puerta de la UE a nuevos mercados en Oriente Próximo y Asia Central. En este sentido, Irán y Turkmenistán prometían ser los mercados más prometedores. Con Irán fuera de juego por la oposición de los EEUU y por las sanciones impuestas por el programa nuclear iraní (y con Iraq envuelto en una pertinaz inestabilidad), Turkmenistán representaba la gran esperanza de Nabucco. A pesar de que en 2008 se confirmó que el país contaba con muchas más reservas de gas natural de lo que se pensaba, la UE parecía claramente haber perdido el juego frente a China. Hasta 2013, el juego parecía girar necesariamente en torno a conseguir que Azerbaiyán participase con sus modestos recursos, para asegurar la viabilidad de Nabucco en un primer estadio, en espera de mayores suministros en un futuro cercano. La decisión de Azerbaiyán del 2013 de decantarse por el TAP, privó a Nabucco de su razón de ser.

Lo que queda en todo caso fuera de duda, es que por lo menos, Azerbaiyán va a contribuir a la diversificación energética de la UE, sirviendo los mercados de Grecia y de Italia. TAP tiene la perspectiva de alcanzar una capacidad máxima de $20 \mathrm{Mmill}$.mc anuales, lo que desde Italia le permitiría extenderse incluso más allá hacia países como Francia o Alemania ${ }^{50}$. Según las noticias más recientes, el consorcio TAP mantiene el calendario para 2015 y espera que la construcción pueda empezar en mayo de este año ${ }^{51}$.

Sin embargo, lo óptimo sería invitar a otros proveedores con mayor capacidad que Azerbaiyán. ¿Podría la UE reactivar Nabucco ${ }^{52}$ Turkmenistán sigue estando potencialmente sobre la mesa. De hecho, ya en noviembre del 2014 Turquía y Turkmenistán alcanzaron un acuerdo marco para el suministro de gas turkmeno a través del futuro TANAP, mientras que una reunión trilateral de ministros de exteriores de Turquía, Azerbaiyán y Turkmenistán tuvo lugar en enero de este año, formato el cual se quiere elevar a nivel de jefes de estado, con la

\footnotetext{
${ }^{46}$ Seznec, Jean-Francois y Mosis, Samer: " The Zohr Gas Field: A Boon for Egypt", Middle East Institute,9 de septiembre 2015, en http://www.mei.edu/content/at/zohr-gas-field-boon-egypt.

47 "Cyprus will soon know if gas discovered in Egypt extends into island's EEZ", Cyprus Mail, 15 de septiembre 2015, en http://cyprus-mail.com/2015/09/15/in-next-days-cyprus-will-know-if-gas-reserve-discovered-in-egyptextends-into-islands-eez/.

${ }^{48}$ Pannier, Bruce: "Could Iranian Gas Be The Solution For Europe?", Oil Price, 24 de febrero 2015, en http://oilprice.com/Energy/Natural-Gas/Could-Iranian-Gas-Be-The-Solution-For-Europe.html.

49 "TAP pipeline to start construction in 2015", Euractiv, 12 de diciembre 2013, en

http://www.euractiv.com/energy/tap-pipeline-start-construction-news-532291.

50 "Consortium Formed To Build Trans-Adriatic Pipeline", RFE/RL, 30 de julio 2013, en http://www.rferl.org/content/tap-gas-pipeline-consortium-azerbaijan/25061672.html.

51 "TAP invites pre-qualification for offshore construction and linepipes", Pipelines International, 4 de marzo 2015, en

http://pipelinesinternational.com/news/tap invites prequalification for offshore construction and line pipes/91358.

52 Bulgaria está interesada en reactivar el gasoducto, contando con la futura producción de gas natural de Azerbaiyán; ver: "Is Nabucco-West Revivable?", Natural Gas Europe, 10 de marzo 2015, en http://www.naturalgaseurope.com/viability-nabucco-west-revival-26549.
} 
primera cumbre planeada para finales de año ${ }^{53}$. El hecho de que desde 2006 se frustrasen tantas expectativas en este sentido nos fuerza a ser prudentes sobre las perspectivas de éxito de tal proyecto. La indeterminación del estatus legal del Mar Caspio representa sin duda alguna el mayor obstáculo, pues es a través de tal mar, con la construcción de un gasoducto a través del Caspio, que se estudia la posibilidad de realizar el enlace con el TANAP.

\subsection{Qatar}

Qatar tiene unas impresionantes reservas en gas natural, pues se trata de las terceras reservas mundiales con $24,5 \mathrm{Bmc}^{54}$, es con datos del 2014 el tercer mayor productor ${ }^{55}$, así como el segundo país exportador de gas natural del mundo ${ }^{56}$. Qatar no debería corregir su política de moratoria sobre producción ${ }^{57}$, y ello representa un elemento esencial para el futuro de la industria gasística del Magreb, vistos los muy bajos costes de producción del gas natural de Qatar. según estimaciones del Centro de Energía de la Universidad de Columbia, en el informe de Jason Bordoff y Trevor Houser, los costes hasta Europa del LNG de Qatar son únicamente de US\$6mmbtu, mientras que el coste del gas convencional de Argelia y Libia es de US\$7mmbtu y US\$8mmbtu ${ }^{58}$. Si Qatar decidiese en el futuro aumentar su producción, Argelia y Libia podrían encontrarse en competición por cuotas de mercado con un temible competidor. Por el momento, tal perspectiva no se vislumbra.

\subsection{Irán}

Quizá la única opción realmente prometedora sea una que quedaba hasta el momento totalmente excluida por razones geopolíticas obvias: Irán. El progreso de las conversaciones sobre el programa nuclear iraní y el deshielo en las relaciones con EEUU resultante ha impulsado a Irán a palpar el terreno. En agosto del 2014, el vice-ministro de energía Ali Majedi ya propuso su país para un futuro suministro a Nabucco, apuntando a las insuficientes reservas que Azerbaiyán podría destinar ${ }^{59}$. Conscientes de las circunstancias políticas que aún dificultan una óptima cooperación energética con Irán, desde Teherán se ha puesto igualmente sobre la mesa otra posibilidad, en la que se hace valer la privilegiada posición geográfica del país: servir de puente a suministros de gas turkmeno ${ }^{60}$. Con estas propuestas Irán pretende devolver Nabucco prácticamente a su punto de partida, cuando aún se discutía en los años 2006 y 2007 tanto posibles suministros de gas natural iraní, como turkmeno. Hay que destacar que sin duda alguna, Irán ofrece una verdadera posibilidad de superar los obstáculos que presenta el cruce del Mar Caspio. De hecho, en el haber de Irán está que ya existen relaciones comerciales tanto con Turquía como con Turkmenistán, para los cuales, desde hace ya varios años, Teherán no es, ni mucho menos, el verdadero agujero negro energético que sí es para la UE.

\footnotetext{
53 "Trans-Caspian Pipeline: A new Round "For" and "Against"", Natural Gas Europe, 9 de marzo 2015, en http://www.naturalgaseurope.com/turkey-turkmenistan-trans-caspian-pipeline-22549.

54 "Statistical Review of World Energy", British Petroleum (BP) (Junio 2015), p. 20.

55 Ibid., p. 22.

${ }^{56}$ Ibid., p. 29.

${ }^{57}$ Kovessy, Peter: "Report: Qatar's natural gas revenue could plunge 34\% by 2026", Doha News, 30 de marzo 2015, en http://dohanews.co/report-qatars-natural-gas-revenue-could-plunge-34-by-2026/.

${ }^{58}$ Bordoff, Jason y Houser, Trevor: "American Gas to the Rescue? The Impact of US LNG Exports on European Security", SIPA Center on Global Energy Policy, Columbia University (Septiembre 2014), p. 31, en http://energypolicy.columbia.edu/sites/default/files/energy/CGEP American $\% 20 \mathrm{Gas} \% 20$ to $\% 20$ the $\% 20$ Rescue $\%$ 3F.pdf.

${ }^{59}$ Pannier, op. cit.

${ }^{60}$ Ibid.
} 
El reciente acuerdo marco alcanzado el 2 de abril del 2015 en la ciudad suiza de Lausana en el marco de las conversaciones $\mathrm{P} 5+1^{61}$ abre la vía para el fin de las sanciones a Irán, y con ello, las perspectivas de desarrollo del sector energético iraní y ulteriores exportaciones de gas natural. Ello podría resolver la paradoja de que un país con un potencial tan elevado, no haya dado el salto desde que los primeros proyectos fuesen discutidos en los años 70, y peor aún, que el país se haya convertido de hecho en un importador neto desde 1997. La paradoja es especialmente sangrante, teniendo en cuenta que Irán posee las segundas reservas mundiales de gas natural, por detrás solo de la Federación Rusa. Ello podría facilitar que el gas natural se pudiese exportar en el futuro hacia Europa a través de Turquía y de Siria.

De hecho, estas dos son las vías que tienen un cierto nivel de desarrollo y para las cuales se proyectan excedentes, cuyo destino más lógico sería precisamente el mercado energético europeo. En el caso de Turquía, que ya importó 8,4Mmill.mca en $2011^{62}$, y que alcanzó 9,69Mmill.mac en 2014, existe un MoU (memorando de entendimiento) desde el 2008, donde se prevé el desarrollo del yacimiento de Pars $\operatorname{Sur}^{63}$; a cambio de la participación turca, se prevé que los volúmenes desarrollados alcancen el mercado energético europeo ${ }^{64}$. Sin embargo, el escepticismo reinante en cuanto las posibilidades de desarrollo, parece verse confirmado en el reciente rechazo por parte de Turquía de duplicar la importación de gas natural iraní ${ }^{65}$. La otra vía en cambio, a través de Irak y Siria, vistas la situación de caos, parece estar por lo pronto fuera de la mesa: un MoU de 2011, preveía un gasoducto con una capacidad de hasta $40 \mathrm{Mmill}$.mca, lo cual superaba el consumo potencial tanto de Irak como de Siria, quedando pues abierta la vía para que hasta $24 \mathrm{Mmill}$.mca pudiesen destinarse al mercado energético europeo ${ }^{66}$. La posibilidad de que parte de gas natural iraní transite por el TAP podría hacerse realidad en el nuevo contexto. Sin embargo, la capacidad libre sería relativamente escasa.

El que circunstancias ajenas a las sanciones de gran parte de la comunidad internacional no puedan anular otras negativas, como las difíciles relaciones entre Turquía e Irán y la terrible situación de guerra e inestabilidad imperante en Irak y Siria, es particularmente problemático para el mercado energético europeo, especialmente a la vista de la existencia del enorme potencial de los sedientos mercados asiáticos. En este sentido, Irán parece estar en una posición bastante favorable para atender la futura demanda tanto del sub-continente indio, como la de China. En contraste con el frustrante avance de otras alternativas, la construcción de un gasoducto hacia Pakistán está en avanzado estado de desarrollo. El gasoducto IPI (IránPakistán-India), que tendría una capacidad inicial de 22Mmill.mca, podría ser expandido hasta los 55Mmill.mca en el futuro. El caso es que desde 2013 la parte iraní está ya completa y existe un contrato firmado, así como acuerdos en cuanto al precio de venta ${ }^{67}$. Aunque la extensión del gasoducto hasta la India sigue estando en duda, la entrada en juego de China puede mejorar las perspectivas, pues se discute igualmente la posibilidad de que parte del gas

\footnotetext{
61 "The Lausanne Framework and a Final Nuclear Deal with Iran", Policy Brief, Arms Control Association, vol. 7, no 6 (14 de abril 2015), en http://www.armscontrol.org/Issue-Briefs/2015-04-14/The-Lausanne-Frameworkand-a-Final-Nuclear-Deal-with-Iran.

${ }^{62}$ Jalilvand, David Ramin: "Iran's Gas Exports: Can Past Failure Become Future Success?", Oxford Institute for Energy Studies, NG78 (Junio 2013), p. 4, en www.oxfordenergy.org/wpcms/wp-content/uploads/2013/06/NG78.pdf.

${ }^{63}$ Ibid., pp. 4-5.

${ }^{64}$ Ibid., p. 5.

65 "Why Turkey Rejects Doubling Iranian Gas Imports", Natural Gas Europe, 16 de abril 2015, en http://www.naturalgaseurope.com/why-turkey-rejects-doubling-iranian-gas-import-23219.

${ }^{66}$ Jalilvand, op. cit., pp. 8-9.

${ }^{67}$ Ibid., p. 9.
} 
natural exportado a Pakistán pueda ser licuado en el puerto de Gwadar en Pakistán. Ello, además, podría abrir la puerta a la financiación de Pekín $^{68}$.

Sin embargo, en el actual contexto de mejora de las relaciones con la Comunidad Internacional tras el Acuerdo de Lausana, las recientes noticias sobre la construcción de terminales de exportación de LNG desde Irán y en concreto, la exportación vía España con el fin de convertir a este país en un eje importador para la UE han hecho renacer las esperanzas sobre Irán como alternativa energética. Se estarían barajando, por parte de la UE, volúmenes de entre 25Mmill.mca y 35Mmill.mca, lo cual convertiría a Irán en un importante suministrador $^{69}$. Esta posibilidad se desveló en la reunión que se mantuvo en Irán recientemente con una delegación española dirigida por José Manuel García-Margallo, ministro de Asuntos Exteriores de España. Si el principal interés por parte de España parecía estar fijado en el restablecimiento de la importación de crudo, del que Irán había sido uno de los principales exportadores hasta las sanciones del $2012^{70}$, pronto quedó claro que lo más prometedor del viaje de la delegación serían las posibilidades abiertas por posibles exportaciones de LNG, para las que España pretendía convertirse en puerta de entrada a toda Europa $^{71}$. Que esto pueda ocurrir realmente es bastante dudoso: ante el desafío de satisfacer una creciente demanda interna, ante la competición con los mercados asiáticos, y más aún, ante la necesidad de inyectar gas natural en sus pozos petrolíferos para mantener la producción $^{72}$, la UE tiene un margen de acción bastante limitado.

\section{Impacto sobre el Magreb}

Manteniendo las prevenciones ya indicadas en la sección de más arriba, en lo que concierne al impacto de futuras exportaciones iraníes de LNG sobre el Magreb, hay que tener en cuenta un dato fundamental que juega a favor de los dos productores de gas natural en el Magreb, es decir, Argelia y Libia. El primer factor viene determinado por la situación política de las

\footnotetext{
${ }^{68}$ Kennedy, Charles: " What The Iran Nuclear Deal Could Mean For Asia", Oil Price, 9 de abril 2015, en http://oilprice.com/Energy/Energy-General/What-The-Iran-Nuclear-Deal-Could-Mean-For-Asia.html.

${ }^{69}$ Steinhauser, Gabriel y Norman, Laurence: "Iran Could Become Major Supplier of Natural Gas to EU", World Street Journal, 13 de septiembre 2015, en http://www.wsj.com/articles/iran-could-become-major-supplier-ofnatural-gas-to-eu-1442155324; con datos de Eurostat, la UE28 importó en 2013 125Mmill.mca de la Federación Rusa, 95,2Mmill.mca de Noruega, 57,3 Mmill.mca de los Países Bajos, 41,5 Mmill.mca de Argelia y 21Mmill.mca de Qatar, con lo que de cumplirse tales predicciones, Irán podría convertirse en uno de los principales clientes de la UE.

70 "España se ofrece a Irán como su 'mejor embajador en Bruselas y Nueva York"', El Mundo, 3 de marzo 2014, en http://www.elmundo.es/espana/2015/09/07/55ed5b98e2704e58268b457c.html.

71 "Spain Ready to Export Iranian Gas to Europe", Natural Gas Europe, 7 de septiembre 2015, en http://www.naturalgaseurope.com/spain-ready-to-export-iranian-lng-to-europe-25321; en todo caso, queda por ver que la opción del LNG sea realmente más apropiada para Irán que seguir apostando por construir un gasoducto hacia Pakistán y la India, vistos los costes de adquisición de la tecnología e infraestructura necesarios para la exportación de LNG en un contexto de bajos precios (Ver: Temizer, Murat: "LNG exports, a dream for Iran: expert", $A A, 23$ de julio 2015, en http://www.aa.com.tr/en/economy/lng-exports-a-dream-for-iranexpert/23586). Se trataría, en todo caso, de un proceso que ocurriría dentro de muchos años, al contrario que para el crudo, en cuyo caso las infraestructuras necesarias está listas para iniciar exportaciones en cuestión de semanas (Ver: Steffanini, Sara y Oroschakoff, Kalina: "The coming Russia-Iran energy axis: Russia tries to gain a foothold, not a competitor", Politico, 21 de julio 2015, en http://www.politico.eu/article/iran-russia-gas-lnggazprom-energy/).

${ }_{72}$ A este respecto, es muy notable el comentario de Jonathan Stern, presidente del Instituto de Oxford para Estudios Energéticos, quien afirmaba que Irán ganaría más con las inyecciones de gas natural en sus pozos de petróleo que exportando esos mismos recursos gasísticos (Ver: Almeida, Isis: "Iran Is No Qatar, Even With World's Second-Biggest Gas Reserves", Bloomberg, 15 de julio 2015, en http://www.bloomberg.com/news/articles/2015-07-14/iran-no-qatar-even-with-the-world-s-second-biggest-gasreserves).
} 
relaciones entre la UE y Rusia. Como ya se indicó en la introducción, fue el significativo empeoramiento de las relaciones con Moscú a raíz de la crisis de Ucrania, y el resurgimiento del discurso de inseguridad energética en relación con Gazprom el que favoreció la gestación de la actual estrategia energética europea y la intención de diversificar las fuentes de suministros de gas natural. Por tanto, el país desfavorecido por esta nueva política habría de ser Rusia y no países como Argelia y Libia. Para este último, ya indicó en su día el Comisario de Energía, Miguel Arias Cañete:

La UE debería establecer nuevas relaciones con los países proveedores y de tránsito, como Turquía y Argelia, y reforzar las antiguas con buenos amigos, como Noruega, Ucrania y los países de la Comunidad de la Energía ${ }^{93}$

Sin embargo, en caso de que una exitosa relación comercial se estableciese entre la UE e Irán como exportador de LNG, cabe considerar la posibilidad de que con el tiempo se redujese el papel de Argelia como suministrador. En este sentido, en el ya mencionado informe de Bordoff y Houser, Argelia (y Libia) tendrían una sustancial ventaja frente a Irán como potencial competidor, basada en razones estrictamente comerciales. Y es que los costes marginales (contando producción, mantenimiento y transporte) de Argelia y Libia resultarían sustancialmente más competitivos que los de posibles exportaciones iraníes. Según nuestros autores, mientras que el gas natural de Argelia tiene un coste marginal de US\$7mmbtu, y el de Libia US\$8mmbtu, el gas natural de Irán alcanzaría hasta US\$14mmbtu ${ }^{74}$. Esta realidad, que por otra parte pone en duda la idoneidad de los recursos iraníes para Europa ${ }^{75}$, debería representar una notable ventaja en el futuro próximo para Argelia y Libia.

Como hemos podido ver en la sección anterior, Qatar no debería aumentar sus exportaciones de gas natural en los próximos años. Ello es una excelente noticia para Argelia, pues el gas natural qatarí es muy competitivo, si bien no significativamente más que el de Argelia. En todo caso, recurriendo de nuevo al informe de Bordoff y Houser, el LNG qatarí tiene un coste de US\$6mmbtu, ligeramente por debajo de los US\$7mmbtu y de los US\$8mmbtu de Libia ${ }^{76}$. En el caso del LNG de Israel, el coste sería de US\$9mmbtu, no significativamente superior, si bien, como ya hemos visto más arriba, las perspectivas de exportación no son excesivamente halagüeñas para la UE, sobre todo frente a la competición del gas natural egipcio. En cuanto a este último, los costes de producción (sin contar con los costes de transporte y licuefacción) habrían de ser de US $\$ 4 m m b t u^{77}$. Parece que Egipto podría ser un competidor potencial, si bien, como ya hemos tenido la ocasión de ver, las perspectivas de ver a Egipto convertirse en exportador son escasas.

\section{Recursos petrolíferos en el Mediterráneo Oriental y Oriente Próximo}

Al contrario que en el caso del gas natural, no parece haber significativos cambios en el panorama petrolífero en la región, en lo que a nuevos yacimientos de refiere. Sin embargo, su influencia sobre el Magreb, en lo que respecta a la caída de los precios del crudo desde hace

\footnotetext{
${ }^{73}$ Arias Cañete, op. cit.

${ }^{74}$ Bordoff y Trevor, op. cit., p. 31.

75 "Iranian Gas to Europe: Years Down the Road", Natural Gas Europe, 17 de septiembre 2015, en http://www.naturalgaseurope.com/iranian-gas-to-europe-years-down-the-road-25467 y "Post Agreement: The Role of Natural Gas in Iran's Energy Future", "Post Agreement: The Role of Natural Gas in Iran's Energy Future", Atlantic Council, 15 de septiembre 2015, en http://www.atlanticcouncil.org/en/events/webcasts/postagreement-the-role-of-natural-gas-in-iran-s-energy-future.

${ }^{76}$ Bordoff y Trevor, op . cit., p. 31.

${ }^{77}$ Seznec y Mosis, op. cit.
} 
un año, es muy notable, ya que las decisiones tomadas en la región determinan el que los precios no se hayan restablecido, con las debidas consecuencias sobre las finanzas de grandes productores como Argelia.

\subsection{Origen de la caída en los precios del crudo}

El año 2014 se presentó con una sorpresa que pocos esperaban entonces: una espectacular caída de los precios del crudo a nivel mundial, y que hasta día de hoy, un año más tarde, parece mantenerse. Esta súbita caída rompió con una tendencia que se venía manteniendo desde inicios del 2011; en la serie histórica que se inicia con tal año, podemos observar cómo, a excepción de los dos picos alcanzados en 2011 mismo y de nuevo un año más tarde, los precios se mantenían dentro de la horquilla de US\$120 y US\$100 por barril (levemente por debajo en una sola ocasión, a mediados del 2012). Después de los precios récord de principios del 2008, cuando el crudo alcanzó el altísimo precio de US\$140 por barril y tras la recuperación de la caída que siguió al inicio de la crisis financiera mundial, cuando el crudo se derrumbó en cuestión de meses hasta alcanzar solo US\$40, parecía que el crudo se había estabilizado en cuotas bastante altas ${ }^{78}$.

Esta estabilidad parecía estar garantizada por la política declarada del principal productor mundial de petróleo con datos del $2009^{79}$, Arabia Saudí: según Riad, el objetivo era que los precios del crudo se mantuviesen en una horquilla de US\$120 a US\$90 por barril, un precio en el que la monarquía saudí se sentía plenamente cómoda: según cálculos realizados en noviembre del 2014 por el diario WSJ, Arabia Saudí necesitaba un precio de US\$106 para equilibrar los presupuestos del $2015^{80}$; los cálculos recogidos por la prensa de los Emiratos Árabes Unidos (EAU) de tres instituciones, el FMI, el Instituto de Finanza Internacional (IFI), así como la Deutsche Bank (DB), eran los siguientes: US\$87,20, US\$109,40 y US\$104,40 Estos precios eran naturalmente satisfactorios para otros miembros de la OPEC, como por ejemplo, Qatar, Kuwait y los EAU, con umbrales para el equilibrio fiscal situados en US\$77, US\$78 y US\$80 respectivamente ${ }^{82}$. Obviamente, otros países, como Libia e Irán, se encontraban en una situación fiscal mucho más delicada, con lo que habrían necesitado precios por encima de US\$120 por barril para equilibrar sus presupuestos: con datos del semanario británico The Economist, Irán necesitaría entre US\$130 y US\$150 ${ }^{83}$. Argelia se encontraría a niveles similares, mientras que en el caso de Libia, dada su crítica situación, se necesitaría hasta US\$180 por barril, siguiendo datos del FMI y Deutsche Bank ${ }^{84}$.

Es por esta razón por lo que la súbita caída que se originó a mediados del 2014 sorprendió a muchos. Visto el interés estrictamente económico por parte de Arabia Saudí por mantener esos precios, pronto se empezó a especular con una posible politización del crudo, a saber, el dejar hundir los precios con el fin de impactar a las finanzas de una serie de países hostiles. En este sentido hay que destacar que la "primera ronda" de caída en el precio

\footnotetext{
78 "Latest Price \& Chart for Crude Oil Brent", Nasdaq, en http://www.nasdaq.com/markets/crude-oilbrent.aspx?timeframe $=10 \mathrm{y}$.

${ }_{79}$ Arabia Saudí produjo en 2014 el 12,9\% de toda la producción mundial, seguido muy de cerca por la Federación Rusa con un 12,7\% y los EEUU con un 12,3\% (BP, op. cit., p. 8).

80 "OPEC: The Cartel is Standing Pat on Production, for Now", World Street Journal, "Argelia", en http://graphics.wsj.com/lists/opec-meeting.

${ }^{81}$ Raghu, M. R.: "How GCC states' break-even oil prices stack up", The National, 24 de agosto 2015, en http://www.thenational.ae/business/energy/how-gcc-states-break-even-oil-prices-stack-up.

${ }_{82}$ Pfeifer, Karen: "Breaking Even, Breaking Down or Going for Broke?", MERIP, 22 de mayo 2015, en http://www.merip.org/breaking-even-breaking-down-or-going-broke.

83 "Black gold deficits", The Economist, 14 de octubre 2014, en http://www.economist.com/blogs/graphicdetail/2014/10/daily-chart-7.

${ }^{84}$ Gurdgiev, Constantin: "Deutsche Bank on Russian 'Break-Even' Oil Prices", Russia Insider, 22 de julio 2015, en http://russia-insider.com/en/business/break-even-oil-prices-russia/ri8872.
} 
respondía estrictamente a cambios en la oferta y demanda internacional, obviamente inesperados: un elemento a destacar que no pasó desapercibido era el que los EEUU hubiese duplicado su producción de petróleo gracias a los recursos de esquistos ${ }^{85}$. Sin embargo, no era el aumento de producción el responsable directo de la caída de los precios, por la simple razón de que el aumento de la demanda en el mercado asiático había absorbido la oferta; en puridad, se podría decir que durante la fase de estabilidad en los precios que precedió a la actual caída, la producción añadida por los EEUU favoreció que estos no aumentasen más. Ahora, sin embargo, con la caída de la demanda asiática (y en menor medida de otros países como Brasil) por enfriamiento de sus economías ${ }^{86}$, nos encontramos con una superproducción que necesariamente se tradujo en menores precios; así, a partir de los US\$114 por barril en julio del 2014, el crudo inició una rápida curva descendente ${ }^{87}$.

Sin embargo, la decisión de la OPEC en su reunión del 27 de noviembre de no reducir la producción para remediar la sobre-oferta existente, de manera que los precios se pudiesen restablecer, consolidó las sospechas de que podría haber motivos políticos detrás. Ciertamente, los "candidatos" no faltaban: por el contexto político de la crisis ya mencionado en la introducción, existía un obvio interés en dañar económicamente a la Federación Rusa (tal y como se hizo con la ronda de sanciones) en represalia a su intervención militar en Ucrania; siendo dependiente en un $50 \%$ de sus presupuestos de los recursos energéticos, principalmente el petróleo, es lógico que la caída del precio del crudo haya impactado duramente en las finanzas rusas. Sin embargo, cabía la posibilidad igualmente de que fuese Irán, gran rival de Arabia Saudí, el que fuese el principal objetivo de los países productores del Golfo. De entre las teorías consideradas en su momento, cabía sin embargo la de una guerra comercial contra el petróleo de esquistos americano, para así empujar fuera del mercado a un rival comercial ${ }^{88}$. Resulta difícil demostrar plenamente cualquiera de estas hipótesis. Sin embargo, numerosos analistas se decantan por teorías más ligadas a imperativos clásicos del mercado del petróleo: la lucha por la cuota de mercado ${ }^{89}$. La explicación más plausible sería, pues, que los principales miembros de la OPEC, ante la caída inesperada de la demanda, habrían decidido luchar por retener cuotas de mercado, para lo que países como Arabia Saudí, con notables colchones financieros, estaban preparados. Ello explicaría por qué

\footnotetext{
${ }^{85}$ Krauss, Clifford: "Oil Prices: What's Behind the Drop? Simple Economics", New York, 5 de octubre 2015, en http://www.nytimes.com/interactive/2015/business/energy-environment/oil-prices.html.

${ }^{86}$ Echagüe, Ana: "Dinámicas comerciales y geopolíticas del nuevo mercado energético", Política Exterior, $\mathrm{n}^{\circ} 71$ (Invierno 2014-2015), en http://www.politicaexterior.com/articulos/economia-exterior/dinamicas-comerciales-ygeopoliticas-del-nuevo-mercado-energetico/.

${ }^{87}$ Entre otros factores que se apuntaban, y que habrían contribuido a esta caída, estaba el refuerzo del dólar en la segunda mitad de 2014 (Ver: Menton, Jessica: "Why Are Oil Prices Falling? Here Are Four Reasons Crude Prices Continue To Trend Lower", International Business Times, 16 de mazro 2015, en http://www.ibtimes.com/why-are-oil-prices-falling-here-are-four-reasons-crude-prices-continue-trend-lower$\underline{1848742}$ ) y las tensiones generadas por la aparición en la escena geopolítica del Estado Islámico en Iraq y Siria (Ver: Chamberlin, Alex: " Must-know: Why crude oil prices have been falling lately", Market Realist, 21 de agosto 2014, en http://marketrealist.com/2014/08/must-know-why-crude-oil-prices-have-been-falling-lately/.

${ }^{88}$ Algo que pareció pasar desapercibido es el interés que Arabia Saudí tiene en productores con alto coste de producción, como son las compañías de los EEUU, tal y como ha explicado el analista Bassam Fatouh: frente a países con bajos costes de producción como Iraq, y que son candidatos susceptibles de tirar los precios a la baja con el fin de ganar cuota de mercado, los productores de petróleo de esquistos son un factor de estabilidad (Ver: Fattouh, Bassam: "Saudi Arabia's Oil Policy in Uncertain Times - A Shift in Paradigm", Oxford Institute for Energy Studies (21 de octubre 2014), en http://www.oxfordenergy.org/2014/10/saudi-arabias-oil-policy-inuncertain-times-a-shift-in-paradigm/). Arabia Saudí tendría muy presente la experiencia negativa de la década de los 80, cuando a pesar de restringir su producción para así mantener los precios, vio cómo otros productores se aprovechaban aumentando su producción con el fin de ganar cuota de mercado; Arabia Saudí habría decidido hundir los precios en 1985 para así recuperar su cuota.

${ }^{89}$ Echagüe, op. cit.
} 
los precios han llegado a hundirse hasta US\$43 por barril en agosto del 2015, encontrándose a día de hoy en US\$48 por barril ${ }^{90}$.

\subsection{Perspectivas futuras del mercado}

Uno de los elementos más susceptibles de coadyuvar al restablecimiento de los precios en el mercado petrolífero sería la salida del mercado de aquellos productores menos competitivos. En este sentido, todas las miradas estaban puestas en el petróleo de esquistos de los EEUU, dado su elevado coste de producción, ligado a una tecnología de extracción necesariamente más costosa ("fracking") que la extracción convencional. El secretario general de la OPEC, Al-Badri, afirmaba así que el gas de esquistos americano dejaría de ser competitivo tan pronto cayese el precio por debajo de los US $\$ 85$ por barril ${ }^{11}$; ese precio se dejó atrás ya en noviembre del $2014^{92}$, por lo que se esperaría que los efectos hubiesen sido devastadores. Si comparamos 2014 con 2013, vemos cómo la producción aumentó de 7,4 millones de barriles diarios a $8,7^{93}$. El comportamiento durante el actual año 2015, periodo en el que podemos ver con mayor fuerza los efectos de la caída de los precios, es el siguiente: se alcanza un pico de producción de 9,5 millones de barriles diarios en la primera semana de julio, iniciándose únicamente entonces una progresiva caída de la producción, que para la tercera semana de septiembre era de 9,1 millones de barriles diarios ${ }^{94}$. Estos datos parecen apuntar a una inesperada resistencia del "fracking" en los EEUU.

En primer lugar, habría que destacar que en el debate sobre los costes marginales de producción del petróleo de esquistos en los EEUU, no todos los yacimientos tienen los mismos costes. En este sentido, las declaraciones de Al-Badri parecían indicar más bien los costes de producción de aquellos más caros. La sub-directora de la Agencia Internacional de la Energía (AEI) destacaba, por el contrario, que la práctica totalidad de los pozos de petróleo de esquistos tenían costes marginales de producción por debajo de los US $\$ 80$ por barril ${ }^{95}$; más aún, afirmaba que el $82 \%$ tenía costes marginales de US $\$ 60$ por barril o incluso menos ${ }^{96}$. Si observamos el cálculo compilado por el WSJ, vemos cómo, efectivamente, las zonas con mayor número de plataformas de extracción, véase, la cuenca pérmica, "Bakken" e "Eagle Ford", tenían costes marginales de US\$75-US\$57, US\$75-US\$60 y US\$65-US\$53 respectivamente ${ }^{97}$. Teniendo en cuenta que los precios actuales se encuentran en US\$48 por barril, prácticamente la totalidad de los pozos deberían encontrarse con pérdidas; efectivamente, muchas torres de perforación han tenido que dejar de operar. Y sin embargo, como se explica en un informe de Clingendael, esto no necesariamente ha repercutido en una significativa bajada en la producción, tal y como se podría haber imaginado: mejora de la cobertura contra riesgos ("hedging"), un aumento de la eficiencia y una reducción de las tasas por perforación por parte de las empresas de servicios han permitido que no fuese hasta abril

\footnotetext{
${ }^{90}$ Nasdaq, op. cit.

91 Smith, Matt: "The US Shale Breakeven Price Debate", Oil Price, 20 de noviembre 2014, en http://oilprice.com/Energy/Crude-Oil/The-US-Shale-Breakeven-Price-Debate.html.

${ }^{92}$ Nasdaq, op. cit.

93 "Crude Oil Production", US Energy Information Agency (EIA), en

http://www.eia.gov/dnav/pet/pet_crd_crpdn_adc_mbblpd_a.htm.

94 "Weekly US Field Crude Oil Production", US Energy Information Agency (EIA), en http://www.eia.gov/dnav/pet/hist/LeafHandler.ashx?n=PET\&s=WCRFPUS2\&f=W.

${ }^{95}$ Falush, Simon: "Oil price slump yet to hit US shale oil production: IEA chief", Reuters, 15 de julio 2014, en http://www.reuters.com/article/2014/10/13/us-shaleoil-energy-breakeven-idUSKCN0I21GG20141013.

${ }^{96}$ Ibid.

${ }^{97}$ Gold, Russell, Ailworth, Erin y Faucon, Benoit: "Energy Boom Can Withstand Steeper Oil-Price Drop", World Street Journal, 29 octubre 2014, en http://www.wsj.com/articles/energy-boom-can-withstand-steeper-oilprice-drop-1414627471.
} 
del 2015 cuando se empezase a estabilizar la producción ${ }^{98}$. En el mismo informe se apunta a que para finales de este año, las caídas habrían de llevar a la producción a caer a niveles similares a los alcanzados a principios de año ${ }^{99}$, es decir, con datos de la IEA, en torno a los 9,1 millones de barriles diarios, si bien hay que destacar que ese nivel ya parece haberse alcanzado en septiembre mismo ${ }^{100}$. El que la caída se haya demorado tanto, sin duda alguna ha influido para que los precios mantuviesen su descenso a lo largo de gran parte de este año ${ }^{101}$.

Sin embargo, no podemos perder de vista la irrupción, o el retorno, más bien, de un nuevo actor en la escena internacional: Irán. Con el acuerdo alcanzado en Lausana y la puerta abierta al fin de las sanciones, asistiríamos al restablecimiento de las exportaciones de crudo de un verdadero coloso energético, cuarto país a nivel mundial en reservas ${ }^{102}$. Manifiestamente, Irán puede trastocar el mercado mundial del crudo, y lo que muchos temen, enconar la pugna por las cuotas de mercado entre todos los productores. De creer las declaraciones realizadas por el ministro del petróleo de Irán, Bijan Zanganeh, su país está dispuesto a retornar al mercado lo antes posible con el fin de recuperar su cuota de mercado, a pesar de la actual situación depresiva; en este sentido, las cifras que se barajan son significativas: el objetivo sería pasar de los actuales 2,8 a 4,2 millones de barriles diarios. Dependiendo de cómo se comporte la producción de los EEUU, podríamos en todo caso contemplar el escenario de que el incremento de producción compensase la pérdida de vigor del "fracking" en los EEUU.

\section{Impacto sobre el Magreb}

En el caso del gas natural hemos podido ver que los nuevos descubrimientos en el Mediterráneo occidental o el posible aumento de producción por parte de Irán deberían tener un impacto relativo, por la escasa posibilidad de que desplacen a Argelia o Libia en el mercado europeo, ya sea por absorción en los mercados internos (véase Egipto e Irán), en

\footnotetext{
98 "Crude Oil Markets in 2015: The Battle for Market Share", Clingendael International Energy Programme (CIEP) (Julio 2015), p. 3, en http://www.clingendaelenergy.com/publications/publication/crude-oil-markets-in2015-the-battle-for-market-share; no hay que perder de vista igualmente que los productores de petróleo de esquistos en los EEUU suelen ser pequeños productores, dependientes de un alto grado de deuda, y que por tanto están obligados a producir, aunque sea a pérdida, con el fin de poder cubrir los pagos por interés (Ver: Cobb, Kurt: "This Is Why A Serious Decline In U.S Shale Plays Is Not Far Away", 24 de julio 2015, en http://oilprice.com/Energy/Crude-Oil/This-Is-Why-A-Serious-Decline-In-US-Shale-Plays-Is-Not-FarAway.html).

99 Ibid

100 "Weekly US Field Crude Oil Production", op. cit.; según datos de la EIA de septiembre del 2015, la producción debería reducirse para 2016 en un 9\% (Ver: Rascouet, Angelina: "IEA Sees U.S. Shale Oil Shrinking in 2016 on Price Slump", Bloomberg, 11 de septiembre 2015, en http://www.bloomberg.com/news/articles/201509-11/iea-sees-u-s-shale-oil-output-shrinking-in-2016-on-price-slump).

${ }^{101} \mathrm{Si}$ la situación se mantiene, no hay que descartar que los productores de petróleo de esquistos en los EEUU acaben finalmente sufriendo las consecuencias; la dependencia del crédito en este sector lo hace muy vulnerable a una depresión sostenida de los precios (Ver: Crooks, Ed: "US Shale Industry Braced for Banckruptcies", Financial Times, 6 de septiembre 2015, en http://www.ft.com/intl/cms/s/0/5974a3ce-52e0-11e5-b029b9d50a74fd14.html), hasta en el aire la idea de estar presenciando una nueva burbuja (Ver: Crooks, Ed: "Shale looks more like dotcom boom than Lehman debt bubble", Financial Times, 6 de mayo 2015, en http://www.ft.com/intl/cms/s/0/b8345a94-f408-11e4-bd16-00144feab7de.html), a pesar de las predicciones optimistas de la AIE, que prevé que la industria vaya a ser capaz de sobrevivir en el marco de unos precios del crudo tan bajos como los actuales (Ver: "US shale oil boom could become next 'dotcom bubble', says Russian oil boss", The Guardian, 10 de febrero 2015, en

http://www.theguardian.com/business/2015/feb/10/us-shale-oil-next-dotcom-bubble-russia-rosneft-igor-sechinopec).

${ }^{102}$ BP, op. cit., p. 6.
} 
otros mercados (véase Asia) o por no ser competitivos en materia de precios (véase Irán), de manera que será Qatar el actor en que los productores del Magreb más hayan de estar atentos. Sin embargo, el panorama del mercado internacional del petróleo es bastante más sombrío, y ello por dos circunstancias determinadas: una ya acaecida, que es la decisión de los países de la OPEC de no reducir su producción en la reunión del noviembre del 2014 y otra previsible, que es el retorno de Irán al mercado del crudo. Peor aún, los bajos precios del crudo impactan notablemente en los precios del gas natural, ya sea directa (en los precios ligados al crudo) o indirectamente (pues los precios spot suelen tener como referencia los anteriores).

\subsection{La reunión de la OPEC de noviembre 2014}

Nuestros dos productores del Magreb, Argelia y Libia, son miembros de la OPEC, donde juegan un papel tan relevante las monarquías del Golfo: Kuwait, Qatar, EAU y sobre todo, Arabia Saudí ${ }^{103}$. En la reunión que mantuvieron los países miembros el 27 de noviembre de 2014 se constituyeron claramente dos frentes: aquellos países favorables a intervenir para corregir los precios y aquellos, defensores del estatus quo; como hemos tenido la ocasión de ver, fue el segundo bloque, liderado por Arabia Saudí, el que logró imponer su criterio y así dejar los precios en caída libre. El segundo bloque, formado por los miembros más solventes, y mejor preparados ante la tormenta de precios se podía permitir tal decisión ${ }^{104}$. Por el contrario, los miembros más pobres del cártel, y más necesitados, en consecuencia, de las aportaciones fiscales de las ventas de petróleo, preferían la corrección de los precios: entre estos estaban, naturalmente, países como Venezuela e Irán, pero igualmente, Argelia. Estos países no solo han tenido que afrontar unos precios negativos para sus economías, sino que tampoco encuentran margen para aumentar su producción, atrapados como están entre los límites impuestos por la OPEC (30 millones de barriles diarios) y la capacidad de Arabia Saudí de aumentar la producción ${ }^{105}$.

Esta decisión, unida a la ya vista resistencia de los productores de los EEUU, podría verse complementada por el aumento de producción de Irán en los próximos meses, con los efectos que ya hemos mencionado. Esto podría asestar un nuevo golpe a Argelia y Libia, si con ello, los precios no se recuperan, o peor aún, bajan todavía más. Irán no tiene interés en precios tan bajos, con lo que es un aliado para los vulnerables productores del Magreb a la hora de renegociar las condiciones actuales en la OPEC; sin embargo, de no lograr modificar la política de la organización, su papel dentro del mercado necesariamente dañará a Argelia y Libia.

Los efectos de la caída de los precios en las economías de los dos principales productores del Magreb son manifiestos. En el caso de Argelia, el escenario ya remite a la terrible experiencia de mediados de los 80 , con las nefastas consecuencias que ello trajo ${ }^{106}$. Además, Argelia se encuentra en una encrucijada en lo que a estabilidad de refiere, con su

\footnotetext{
103 "OPEC Member Countries", en http://www.opec.org/opec web/en/about us/25.htm.

${ }^{104}$ Lawler, Alex, Sheppard, David y El Gamal, Rania: "Saudis block OPEC output cut, sending oil price plunging", en http://www.reuters.com/article/2014/11/28/us-opec-meeting-idUSKCN0JA0O320141128.

${ }_{105}$ Critchlow, Andrew: "Opec unity cracks as disgruntled members call for meeting to stem oil slump", The Telegraph, 20 de agosto 2015, en http://www.telegraph.co.uk/finance/oilprices/11814760/Opec-unity-cracks-asmembers-call-for-meeting-to-stem-oil-slump.html.

${ }^{106}$ Ghilès, Francis: "Algeria Buffeted by Falling Oil Prices and Growing Social Unrest", CIDOB, no 342 (14 de julio 2015), en

http://www.cidob.org/en/publications/publication_series/opinion/mediterraneo y oriente medio/algeria buffete d by falling oil prices and growing social_unrest; Escribano, Gonzalo: "La economía argelina acusa el golpe", Blog Real Instituto Elcano (16 de abril 2015), en http://www.blog.rielcano.org/la-economia-argelinaacusa-el-golpe/.
} 
vecina Libia sumida en el caos y con el área del Sahel representando un peligro constante ${ }^{107}$; la inestabilidad en Argelia si la economía se resintiese por los actuales precios del crudo sería muy negativa a nivel regional. Tanto o más puede decirse de Libia, país que se encuentra en una situación de caos por la guerra de facciones en que se sumergió tras la caída de Gaddafi.

\section{Conclusiones}

¿Qué efecto tendrá la revisión de la estrategia energética de la Unión Europea mencionada al inicio para los países del Magreb? Este artículo se ha centrado en los efectos de los cambios en el Mediterráneo oriental, así como Oriente Próximo, en lo que a gas natural y petróleo se refiere, al tratarse estas dos fuentes de energía en las principales exportaciones de los países productores del Magreb. Sin embargo, la introducción referida a la política energética de la UE es pertinente, pues es un factor que puede entrar en conjunción con los cambios en las dos regiones mencionadas en este párrafo.

En este sentido, hay que destacar que los cambios en la política energética europea conciernen principalmente a la diversificación del gas natural, y no tanto el petróleo. Ello es por una razón sencilla: el gas natural contiene mayores factores de vulnerabilidad, dada la alta dependencia de la Unión Europea de gasoductos; al contrario, el suministro de petróleo depende principalmente del transporte marítimo, menos vulnerable a cortes ocasionales. Es por ello, que por encima de la reducción del consumo de hidrocarburos en general, por razón de la lucha contra el cambio climático, y que aquí no tratamos, la diversificación de fuentes de gas natural se convierte en uno de los elementos capitales de la nueva política energética. Sin embargo, los países suministradores del Magreb, Argelia y Libia no son el objetivo de esta política de diversificación, sino Rusia, en el punto de mira desde el inicio de la crisis en Ucrania.

Es por ello que se puede concluir de la filosofía misma de la nueva estrategia energética que tal política de diversificación no debería afectar al Magreb. Sin embargo, hemos analizado si podría afectar indirectamente. Es por ello que hemos pasado a considerar la previsible evolución de los recursos energéticos en el Mediterráneo oriental y Oriente Próximo, pues representan nuevos recursos potenciales que podrían verse aprovechados por la Unión Europea en su política de diversificación. La cuestión fundamental aquí ha sido la de determinar si los nuevos recursos ofrecerían unos precios más competitivos que los recursos del Magreb, sentando las bases de una mayor competición en el futuro, desfavorable para su posición de mercado. Parece razonable concluir, que: será difícil que la UE pueda ser receptor de muchos de los nuevos recursos, ante $1 \%$ la importancia de los mercados internos de los países productores (véase, Egipto e Irán) y $2^{\%} /$ la competición de mercados internacionales (véase, por ejemplo, el mercado asiático). Si aun así, parte de los recursos estuviesen disponibles, una constatación de la competitividad de los precios del gas natural de Argelia y Libia hace suponer que estos países vayan a retener sus cuotas de mercado sin demasiada dificultad.

El panorama con el segundo de las fuentes de hidrocarburos, el petróleo, es doblemente diferente: por el escaso impacto, en cuanto a diversificación, de la nueva política europea, pero por el muy sustancial impacto de, sobre todo, Oriente Próximo sobre el Magreb. Esto se debe a que las decisiones tomadas en el seno de la OPEC, por impulso de las monarquías del Golfo, y especialmente Arabia Saudí, de no reducir la producción ante la caída de la demanda

${ }^{107}$ Véase, por ejemplo, Boukhars, Anouar: "Es hora de replantear la seguridad en el Sáhara y el Sahel", Policy Brief, FRIDE (8 de abril 2015), en http://fride.org/publicacion/1257/es-hora-de-replantear-la-seguridad-en-elsahara-y-el-sahel. 
que impulsó hacia abajo los precios, han provocado una mayor caída si cabe. Ello impacta muy negativamente en los países productores del Magreb, como Argelia y Libia, menos preparados que Arabia Saudí, por ejemplo, para soportar de forma sostenida precios tan bajos. A ello se añade el fin de las sanciones a Irán y su previsible retorno a nivel internacional como exportador de crudo; si con ello aumenta la producción, compensándose la previsible caída en la producción de petróleo por parte de los EEUU, será un factor añadido que coadyuve al mantenimiento de la presión bajista, con las consecuencias que ello tendrá para las finanzas de los productores del Magreb. 